\title{
Canine Obesity: An Inflammatory Disease Related To Oxidative Stress
}

\author{
Victor Gerardo Petro Hernández, Veridiane da Rosa Gomes*, Luma Tatiana Silva Castro, Naida Cristina Borges and \\ Maria Clorinda Soares Fioravanti
}

Department of Veterinary Medicine, Federal Univesity of Goiás, Brazil

*Corresponding author: Veridiane da Rosa Gomes, Department of Veterinary Medicine, School of Veterinary Medicine and Animal Science, Federal Univesity of Goiás-UFG, Campus Samambaia, Avenida Esperança, s/n, Conj. Itatiaia, Goiânia, GO 74690-900, Brazil

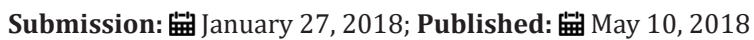

\begin{abstract}
Obesity is a big concern in dogs since it's an epidemic condition increasing last decades. The disease is defined as the excessive storage of fat in the body by due the consumption of large amounts of food. The over food intake or inadequate energy usage may cause a positive energy balance. Several factors may predispose dogs to obesity such as breed, age, genetics, sex, reproductive status, diet, environment, dog's physical activity and owner habits may be related. In addition, it is linked to decreased longevity and several other diseases such as cardiomyopathy, chronic kidney disease, diabetes mellitus, hyper adrenocorticism and hypothyroidism. The fat tissue triggers an inflammatory response, activating immune cell response to produce inflammatory cytokines and oxygen reactive species increasing oxidative stress. Due the importance and the impact this disease has on the animal's quality of life, this systematic review aims to contribute describing main mechanisms of the inflammatory process of adipose tissue in obesity.
\end{abstract}

Keywords: Endocrinopathy; Cytokines; Inflammation; Little animals; Free radicals; Obesity; Oxidative stress

\section{Introduction}

Obesity has been an important topic concerning dog's health, being recognized as an important disease worldwide due it's epidemic character [1,2]. According to the US Association for Pet Obesity Prevention (APOP), the prevalence of obesity in dogs was 55\%, 52\%, 54\% and 54\% in 2010, 2014, 2015 and 2016, respectively [3]. In Brazil, a study revealed the prevalence of $17 \%$ of obese animals in a total of 648 dogs in the city of São Paulo [4]. Other Brazilian studies from the state of Pernambuco and Rio Grande do Norte interviewed 167 veterinarians to determine obesity among their patients giving an overall prevalence of $28.5 \%$ in the year 2012 [5]. Obesity is defined due to the excessive accumulation of adipose tissue in the body. It may accumulate by ingesting large amounts of food or inadequate energy usage, which causes a positive energy balance [1]. Obesity is related to the development of metabolic diseases such as metabolic syndrome, type 2 diabetes, cardiovascular disease $[6,7]$ and inflammation of the adipose tissue itself [6].

The inflammation of adipose tissue plays an important role in the production of cytokines, such as interleukins (IL-1, IL-6) and tumor necrosis factor (TNF). These products stimulates the production of C-reactive protein [6] and oxygen reactive species, which lead to oxidative stress $[8,9]$. The knowledge of obesity and its system alterations primarily are crucial to understand its pathophysiology and treatment and prevention.

\section{Literature Review}

\section{Adipose tissue}

Adipose tissue is composed of several cell types such as preadipocytes, adipocytes, mesenchymal stem cells, endothelial, nerve and defense cells. This fat cell consists in $50 \%$ of all cellular content [10]. It presents precursors with phagocytic capacity that can be transformed into macrophage-like cells in response to appropriate stimuli [11].

In mammals, there are two types of adipose tissue, white adipose tissue (WAT) and brown adipose tissue (BAT). A single large lipid droplet of mature WAT occupies $85-90 \%$ of the cytoplasm, and displaces the nucleus in a small part of the cytosol to the periphery. The BAT is specialized in thermo genesis, therefore, it participates in the regulation of body temperature in foetuses and newborns, being absent in adult humans. It can reach $60 \mu \mathrm{m}$ in diameter and has several cytoplasm lipid droplets of different sizes, abundant cytoplasm, spherical and eccentric nucleus, large number of mitochondria, which use the energy released by the oxidation of metabolites, mainly fatty acids, to generate heat [12]. 
The TAG is directly related to the amount of accumulated fat in body (Pond 2011). The fat storage is distributed in several deposits, anatomically classified as subcutaneous adipose tissue (SAD) and visceral adipose tissue (VAT). The SAD is represented by deposits under the skin in the abdominal, gluteal and femoral regions. The VAT is deposited near or even within the viscera of the abdominal cavity, such as mesenteric, omental and retroperitoneal fat [13].

\section{Adipose tissue alterations in obesity}

Obesity triggers an inflammatory response in adipose tissue in animal models [6]. The lean fat expresses M2 markers in the macrophage surface that can be alternatively activated. On the other hand, obesity leads to the recruitment of T cells in the adipose tissue and the accumulation of M1 markers of macrophages that can be classically activated $[6,7,14]$. Both, they produce excessive inflammatory cytokines [15].

Under physiological conditions, the adipose tissue produces pro-inflammatory and anti-inflammatory cytokines called the adipocytokines or adipokines. They are secretory bioactive substances affecting near and distant organs. Most of these adipocytokines are pro-inflammatory and a small number of adipocytokines have anti-inflammatory properties [15]. They seem to be crucial for homeostasis related to the nutritional status [7]. Thus, there is macrophage aggregation, inflammation and hyperplasia of fat tissue that can prevent hypertrophy, cell necrosis and inflammation [16]. However, the adipose tissue may not undergo substantial hyperplasia, presenting lower inflammation response [6]. Consequently, obesity is associated with low-grade inflammation characterized by increased infiltration of adipose tissue macrophages and production of inflammatory cytokines [6].

Abnormal adipose tissue growth may lead to deregulation, increasing the production of adipocytokines, including leptin, TNF-a, IL-6, PAI-1, MCP-1, angiopoietin 2 (Angptl2) protein, promoting metabolic and cardiovascular diseases. On the other hand, beneficial adipocytokines such as adiponetine, which have anti-inflammatory properties, are highly produced by lean adipose tissue and its production is reduced in obesity. Thus, the production of pro-inflammatory and anti-inflammatory adipocytokines imbalance in obesity contributes to the development of obesityrelated disorders [6,17].

Adipocytes from obese patients have a lower density of insulin receptors and a greater density of beta- 3 adrenergic receptors, thus increasing the rate of lipolysis with release of free fatty acids, leading to various metabolic disorders such as increased production of radicals free insulin-induced induction of insulin resistance and synergism in the action of IL-6 and TNF-a, causing induction of apoptosis in pancreatic beta cells. Taken together, these effects are classified as lipotoxicity [18].

Lipotoxicity causes anatomical and functional lesions in different cell types. Adipose tissue dysfunction and lipotoxicity comprise two mechanisms that explain pro inflammatory status and insulin resistance (IR) [9]. Obese mices shown a marked increase of this expression was found in adipocytes, providing binding with insulin resistance. The TNF-a may stimulate the cellular kinase complex known as I Kappa B Kinase (IKK), which activates nuclear factor (NF), a transcription factor that, in turn, boosts the production of pro inflammatory cytokines including IL1b, IL-6, TNF and interferon [18].

\section{Main pro-and anti-inflammatory adipokines in obesity}

For several decades, adipose tissue was considered as an inert tissue which main the function was to storage fat in the form of triglycerides (TG), being designated a passive organ [19]. However, it's been recognized now that it is endocrine organ metabolically active thanks to the discovery of leptin synthesized exclusively by adipose tissue adipocytes [20].

More than 50 adipokines, cytokines, chemokines and other mediators are produced by adipose tissue, which play a role in the control of glucose and lipid metabolism, blood pressure, insulin resistance and others [18]. Moreover, adipokines are involved in vascular homeostasis (plasminogen activator inhibitor 1 PAI-1), glycemic homeostasis (adiponetin) and angiogenesis (vascular endothelial growth factor - VEGF) [21].

Tumor necrosis factor- $\alpha$ (TNF- $\alpha$ ): The TNF- $\alpha$ is a proinflammatory cytokine produced by monocytes and macrophages in muscle, adipose and lymphoid tissue [18,22]. It plays a central role in inflammatory and autoimmune diseases. In 1993, TNF- $\alpha$ was identified as a pro-inflammatory adipocitocin potentially involved in the pathogenesis of insulin resistance [18], by decreasing GLUT-4, phosphorylation of insulin receptor substrate 1 (IRS-1), and specific insulin receptor phosphorylation [22] in muscle and adipose tissues [23].

The TNF- $\alpha$ may affect the uptake of insulin-stimulated glucose in obese rodents, reducing the use of glucose by fat, muscle, or both. Although this may occur through circulating TNF- $\alpha$, it is also possible that local secretion into adipose deposits or into adipose tissue that permeates the muscles of obese animals may inhibit insulin-dependent uptake of glucose [18].

In addition to high production of TNF- $\alpha$ by adipose cells or per RNA unit, obese and human animals have an excessive amount of adipose tissue which often includes a high number of fat cells. This could exacerbate overproduction of TNF-Î \pm , whether the effects are achieved locally or systemically [18].

The TNF- $\alpha$ activates the nuclear factor $\kappa B(N F-\kappa B)$, resulting in increased expression of adhesion molecules on the surface of endothelial cells and vascular smooth muscle cells, resulting in an inflammatory state in adipose tissue, endothelial dysfunction and atherogenesis [24].

reactive protein (CRP): The C-reactive protein is a positive acute phase molecule produced in the liver and adipose tissue in response to internal and external lesions. It binds to neutrophil membrane receptors and mononuclear cells. It stimulates the release of pro-inflammatory cytokines [25]. 
The PCR is a mediator of innate immunity, which binds to an Fc $\gamma$ RI1 receptor for acquired immune response, with an affinity comparable to that of IgG. During the acute phase response, elevated levels of circulating CRP may influence IgG complex signaling by Fc $\gamma$ RII [25].

The PCR is a potent stimulator of tissue factor production by macrophages, activating the complement system in vivo, binding to lipoproteins, such as LDL and VLDL, facilitating its aggregation. In addition, CRP is expressed by monocytes, accumulating in the incipient atherosclerotic lesions in the aorta and coronary arteries. Data from the literature suggest a direct participation of CRP at the beginning or progression of the atherosclerotic lesion [26].

4.3.3. Leptin: The Leptin is a protein composed of 167 amino acids produced in adipose tissue [27], mammary gland, skeletal muscle, gastric epithelium and placental trophoblast [28]. It's acts controlling alimentar ingestion due to its action on neuronal cells of the hypothalamus in the central nervous system, promotes the reduction of the alimentary intake; increases energy expenditure; regulates the neuroendocrine function and the metabolism of the glucose and of fats [28] to from the activation of specific receptors in the target organs such as the long-chain ObRb receptors with greater expression in the hypothalamus and the short-chain ObRa receptors found in the pancreas and more specifically in the $\alpha$ and $\delta$ cells of the islets of Langerhans [29].

This protein reduces appetite by inhibiting the formation of appetite-related neuropeptides, such as the neuropeptide $\mathrm{Y}$ The other path is by increasing the expression of anorexigenic neuropeptides ( $\alpha$-MSM), corticotropin releasing hormone (CRH) and substances synthesized in response to amphetamine and cocaine. High levels of leptin may reduce food intake while low levels of this substance may induce hyperphagia [28]. The leptin can also inhibits lipogenesis and stimulates lipolysis by reducing intracellular lipid levels in skeletal muscle, liver and pancreatic beta cells, thereby improving insulin sensitivity. The limbic system stimulates the reabsorption of dopamine, thus blocking the pleasure of eating and, through the nucleus locus coeruleus, activates the sympathetic nervous system, which leads to an increase in resting energy expenditure [9]

This protein also protects T-lymphocytes from apoptosis and regulates the proliferation and activation of $\mathrm{T}$ cells. Leptin also influences the production of cytokines from $\mathrm{T}$ lymphocytes, generally altering the phenotype for a TH1 response [30] the activation of monocytes, phagocytosis and the production of cytokines, promoting oxidative stress and vascular inflammation, besides the stimulation of the proliferation and migration of endothelial cells and smooth muscle cells, thus favoring the development of atherosclerosis [31,32].

In inflammation, leptin acts directly on macrophages to increase phagocytic activity and the production of proinflammatory cytokines. It also exerts an effect on T cells, monocytes, neutrophils and endothelial cells. Increased levels of C-reactive protein (CRP) are produced, thus proving its inflammatory effect when leptin is administered [32].

The production of leptin is controlled by substances such as insulin, glucocorticoids and pro-inflammatory cytokines. Infectious states and endotoxins may increase plasma leptin concentrations. The catecholamines can reduce their production in cold temperatures and due to testosterone production. Stress conditions, such as prolonged fasting and intense physical exercises may lead to a decrease in the circulating levels of leptin. This shows how central nervous system to inhibit the release of leptin by adipocytes [29].

There are mechanisms of resistance to the action of leptin, either by problems in their receptors or defects in the passage of the hormone through the blood-brain barrier. As a result of the inability of the leptin action, the satiety center is not activated and maintenance of the positive energy balance, with greater accumulation of fat occurs. As a result, the body develops hyperleptinemia associated with leptin resistance, binding this hormone to its peripheral receptors, mainly allocated to the heart and endothelial cells [33].

There are 3 general ways in which changes in leptin regulation could lead to obesity:

a) Inability to produce leptin, which would result in obesity.

b) Low leptin secretion.

c) Relative or absolute insensitivity to leptin at its site of action.

The lack of receptors or defects in the passage of the hormone through the blood-brain barrier, the increase in circulating leptin, analogous to the increase in insulin levels may be observed with insulin-resistant diabetes and also hyperleptinemia associated with leptin resistance. This may resulting in the binding of this hormone to its peripheral receptors, mainly allocated to the heart and endothelial cells $[28,34]$. Differences in leptin production and leptin sensitivity may be the result of genetic, environmental and psychological factors [28].

Plasminogen activator inhibitor-1 (PAI-1): The Plasminogen activator inhibitor-1 (PAI-1) is regulated in deposits of visceral adiposity during the development of fat accumulation in a rat obesity model. Plasma levels of PAI-1 correlate positively with visceral adiposity in humans. PAI-1 may serve as a thrombogenic adipocytokine that potentially contributes to the pathogenesis of obesity-related thrombotic disorders [35]. It may regulate blood supply of adipose tissue and the flow of fatty acids from it $[36,37]$. Plasma levels of PAI- 1 are regulated by the accumulation of visceral fat, and a high concentration of PAI-1 is associated with insulin resistance, as well as pro-inflammatory cytokines [36].

Adiponetine ACRP30: Adiponectine ACRP30 was found as a specific adipocytokine and its expression is unexpectedly decreased in obesity [38,39]. It is a protein structurally homologous 
to collagens VIII and X and the complement system factor $\mathrm{C} 1 \mathrm{q}$ expressed and secreted exclusively in differentiated adipocytes [37]. The ACRP30 regulates energetic homeostasis, glucose and lipid metabolism and anti-inflammatory action [40]. The antiinflammatory and antiatherogenic function because it inhibits the adhesion of monocytes to endothelial cells, the transformation of macrophages into foam cells and activation of endothelial cells, inhibits the expression of TNF- $\alpha$ [1], decreases CRP levels and increases nitric oxide (NO) in endothelial cells [41].

Adiponetine improves insulin sensitivity, decreases the flow of free fatty acids and increases their oxidation, inhibits the major gluconeogenic liver enzymes, reduces hepatic glucose and muscle release, and stimulates the use of glucose and fatty acid oxidation [23]. In addition, its globular isoform inhibits cell proliferation and ROS production induced by low-density lipoprotein (LDL) oxidase during atheromatous plaque formation [42].

High levels of adiponetine usually lead to weight loss. Adiponetin gene polymorphisms have also been associated with obesity and insulin sensitivity [40]. Adiponetine deficiency results in the reduction of NO in vascular walls and promotes leukocyte adhesion, causing chronic vascular inflammation41 and its inhibition and is given by TNF- $\alpha$ and IL-6 [10].

Interleukin 6: Interleukin-6 is a cytokine produced by macrophages and adipocytes [32]. This is a cytokine that may defense to inflammation and tissue damage [9] and by cells of the immune system, fibroblasts, endothelial cells and skeletal muscle [10].

Circulating levels of IL-6 correlate with body mass index (BMI), insulin resistance, and carbohydrate intolerance Friedman and Halaas [28]. IL-6 also influences glucose tolerance through down regulation of visfatin. In addition, it antagonizes the secretion of adiponectin [9] and in animal models, elevates TG levels, increasing gluconeogenesis and glycogenolysis and inhibiting glycogenesis [42].

Resistant: Resistin (RSTN) is an adipokine produced by mature adipocytes and macrophages, and may be the link between obesity and insulin resistance [37]. It has hyperglycemic properties; levels of circulating resistin are proportional to the degree of adiposity [10]. It is linked to the inflammatory environment due to its predominant monocyte production and its correlation with IL-6 levels [9].

RSTN is a hormone that potentially binds obesity with diabetes, since in type 2 diabetes mellitus (T2DM), characterized by resistance of the target tissue to insulin, levels of circulating resistin are decreased by the anti-diabetic drug rosiglitazone and are increased in induced and genetic dietary forms of obesity. Administration of the anti-resistin antibody improves the action of blood sugar and insulin in mice with diet-induced obesity. In addition, treatment of normal mice with recombinant resistin impairs glucose tolerance and insulin action. Insulin-stimulated glucose uptake by adipocytes is increased by the neutralization of the resistin and is reduced by treatment with resistin [43].

\section{Oxidative stress}

Oxidative stress is the imbalance between oxidant molecules and antioxidants that results in the induction of cellular damage by free radicals (RL), and RL are also known as reactive oxygen species (ROS) highly reactive and unstable chemical molecules. their composition has an unpaired electron in its outermost orbit, and to remain stable they must donate or withdraw an electron from another molecule $[33,44]$.

Free radicals can be generated in the cytoplasm, in the mitochondria or in the membrane under physiological conditions and in many diseases. Its cellular target proteins, lipids, carbohydrates and DNA are related to its formation site. Some species of free radicals are $[45,46]$ :

a. $\quad 02$ - superoxide radical

b. $\quad \mathrm{OH}$ - hydroxyl radical

c. NO - nitric oxide

d. ONOO- peroxynitrite

e. Q - Semiquinone radical

It may cause peroxidation of the fatty acids constituting the double lipid layer. Thus, oxidative stress (EO) is involved in different pathological processes, among them obesity [46]. Obesity promotes an increase in plasma lipid peroxidation, which is a process that generates free radicals that occurs in each cell membranous structure [47].

Lipolysis increased is due to the excessive release of nonesterified fatty acids. Intracellular increase of fatty acids and diacylglycerols (DAG) activate the protein kinase C (PKC), which stimulates the NADH oxidase enzyme, which increases the production of oxygen reactive species and inhibits the production of nitric oxide (NO) $[48,49]$.

\section{Mechanisms of free radical formation in obesity}

Adipose tissue: The increase in oxidative stress associated with obesity is due to excess adipose tissue, since adipocytes and pre-adipocytes have been identified as a source of proinflammatory cytokines that are potent stimulators for the production of oxygen and nitrogen reactive species by macrophages and monocytes. TNF- $\alpha$ also inhibits PCR activity, increasing the interaction of electrons with oxygen to generate superoxide anion [9].

Adipose tissue also has the secretory capacity of angiotensin II, which stimulates the activity of nicotinamide adenine dinucleotide phosphate (NADPH) oxidase. NADPH oxidase comprises the main route for the production of ROS in adipocytes [50].

Oxidation of fatty acids: Inflammation is the primary mediator of hepatic cell injury, where oxidative stress is secondary to the release of inflammatory mediators, such as reactive oxygen 
species (ROS) and nitro radicals. There are three pro-oxidant ROS generating sites in the hepatocytes: mitochondria, peroxisomes and smooth endoplasmic reticulum, which, in cell fractionation, form the microsomes, therefore, EO, which could result in mitochondrial DNA alterations in the oxidative phosphorylation that occurs in the mitochondria, causing structural abnormalities and depletion of adenosine tri-phosphate (ATP). However, it is also possible that mitochondrial abnormalities are pre-existing conditions that allow the overproduction of ROS in the improved fatty acid beta oxidation scenario [51].

Excessive oxygen consumption: Excess fat increases the mechanical load and myocardial metabolism, therefore, oxygen consumption is increased leading to the production of ROS as superoxide, hydroxyl radical and hydrogen peroxide derived from increased mitochondrial respiration and the loss of electrons produced in the chain of the electron transport, resulting in the formation of Superoxide [47,52,53].

The nutritional obesity linked to the consumption of hyperlipidemic diets are involved in the metabolism of oxygen. Fatty acids have double bonds in the molecules that are vulnerable to oxidation reactions and consequently cause lipid peroxidation [54].

Accumulation of Cell Damage: Obesity can cause progressive and cumulative cell damage that result from high body mass pressure. Cell injury causes the release of cytokines, especially tumor necrosis factor alpha (TNF- $\alpha$ ), which generates ROS from tissues, which in turn cause lipid peroxidation [8].

Antioxid: The concentration of oxygen-reactive species within the cells is extremely low because of the antioxidants that remove them or prevent their formation; they may be enzymatic and nonenzymatic antioxidants [55]. Antioxidant defense systems eliminate and minimize the formation of oxygen-derived species, but they are not $100 \%$ effective. Thus, antioxidants may be particularly important in reducing cumulative oxidative damage and helping the individual to remain healthy for longer [44]. Obesity has been associated with a low-grade pro-inflammatory state in which changes in oxidative stress and antioxidant mechanism may be involved [56].

In humans, studies show that reduced levels of vitamin $\mathrm{E}$ in the tissue are associated with obesity [54], and a reduction in the vitamin E content of the membrane, compromises the ability of the cell to defend against hydroxyl radicals [57]. Another study found that levels of serum antioxidants, such as vitamin E, vitamin $C$ and $\beta$-carotene, as well as glutathione, are reduced by obesity [58].

Obesity persistance, antioxidant sources can be depleted by decreasing the activity of enzymes such as superoxide dismutase (SOD) and catalase (CAT) [47].

In obesity, plasma levels of C-reactive protein, interleukin-6, tumor necrosis factor, and lipid peroxides are elevated, whereas endothelial nitric oxide levels are low because of low-grade systemic inflammation, as well as plasma and tissue activities (AChE) and butyrylcholinesterase (BChE) enzymes are high and as a result of this increase plasma and acetylcholine (ACh) tissue levels will be low [59].

The AChE and BChE have been identified as low grade inflammatory markers. Several studies have shown that acetylcholine (ACh), increased plasma, leukocytes, red blood cells, platelets and other tissue concentrations of AChE and BChE enzymes reflect indirectly by reducing acetylcholine concentrations by increasing local and systemic inflammation [59].

Oxidative stress generated by obesity complications: Obesity and the consequent SO production has been associated with the development of other pathologies [42]:

a. Diseases associated with obesity.

b. Insulin resistance and diabetes

c. Systemic arterial hypertension

d. Ischemic heart disease

e. Obstructive sleep apnea, asthma

f. Gout

g. Peripheral vascular disease

h. Problems of psychology (social stigmatization)

i. Rheumatic and orthopedic problems

j. Oncology problems

k. Hepatic insufficiency

Obesity may lead to the development of non-alcoholic steatohepatitis, which appears as a result of the circulating in AGL that are released by adipose tissue in response to insulin resistance. The amount of fatty acid free FFA is proportional to plasma; in addition, it also increases lipogenesis in the body and improves intracellular accumulation of fat. This last one is the first step in the development of non-alcoholic fatty liver disease, while the second step is inflammation and cirrhosis [60].

The dysfunction of adipose tissue, as it occurs in obesity and insulin resistance, is characterized by the activation of an inflammatory signal. Some of these signs arise, directly or indirectly, from secreted substances in adipose tissue. EROs are generated in places of inflammation and damage; A high concentration of these can cause cell damage and death, and specifically, EO increases vascular endothelial permeability and promotes leukocyte adhesion $[61,62]$.

LDL oxidation is also associated with an increase in the expression of adhesion molecules in the endothelium, facilitating the infiltration of monocytes into the subendothelial space [63], the role of adipose tissue and its secretory adipokine as a major causes of endothelial dysfunction have been emphasized [61]. 
There is a higher content of superoxide radicals and nitrotyrosine in the coronary endothelium in obese dogs. Early obesity is characterized by increased OS and endothelial dysfunction associated with increased leptin levels [64]. The weight loss improves endothelium-independent vasodilation, markers of endothelial activation and decreases proinflammatory cytokine levels [61].

\section{Conclusion}

Adipose tissue is a secretory organ of great importance to the organism. The ability of secrete substances is a specific biological function. Obesity is characterized by excessive storage of adipose tissue, increased secretion of adipokines. Therefore, the effects produced in the body may change and leptin resistance may occur.

In addition to adipokines, the overproduction of oxygen reactive species (ROS) may trigger damage cellular along with the subproduction of nitric oxide (NO). Progressive accumulation of fat is related to the development of other diseases in dogs.

Risk factors may interact with the immune system, resulting in an inflammatory process triggered by oxygen-reactive species (ROS) that become harmful if perpetuated.

Several immune-pathological processes are being investigated, allowing new therapeutic targets to reduce the cascade of proinflammatory signalling. Obesity also causes systemic oxidative stress in dogs and full knowledge of the mechanisms may guide new strategies for the prevention of associated systemic diseases.

\section{References}

1. German AJ (2006) The growing problem of obesity in dogs and cats. J Nutr 136(7Suppl): 1940S-19466S.

2. German AJ (2016) Weight management in obese pets: the tailoring concept and how it can improve results. Acta Vet Scand 58(1): 57.

3. Calabash N (2016) Fat Pets Getting Fatter According To Latest Survey. Association for Pet Obesity Prevention. Amirkhizi F, Siassi F, Minaie S Djalali M, Rahimi A, Chamari M (Eds.), (2010) Is obesity associated with increased plasma lipid peroxidation and oxidative stress in women? ARYA Atheroscler 2(4): 189-192.

4. Jericó M, Scheffer K (2002) Epidemiological Aspects of Obese Dogs in the city of São Paulo. Clín Vet 7(37): 25-29.

5. Neves IV, Souza TA, Moura AC, Lima JYB, Cardoso MJL, et al. (2013). Veterinary Obesity Questionnaire on Dogs. In: 34o Anclivepa Brazilian Congress, NATAL, Anais - II Acta Veterinaria Magazine.

6. Chandalia M, Abate N (2007) Metabolic complications of obesity: inflated or inflamed? J Diabetes Complications 21(2): 128-136.

7. Ouchi N, Ohashi K, Shibata R, Murohara T (2012) Adipocytokines and obesity-linked disorders. Nagoya J Med Sci 74(1-2): 19-30.

8. Khan N, Naz L, Yasmeen G (2006) Obesity: an independent risk factor for systemic oxidative stress. Pak J Pharm Sci 19(1): 62-65.

9. Fonseca MHA, Takada J, Alonso-Vale MIC, Lima FB (2007) Adipose tissue as an endocrine organ: from theory to practice. J Pediatr (Rio J) 83(5): S192-S203.

10. Sánchez-Muñoz F, García-Macedo R, Alarcón-Aguilar F, Cruz M (2005) Adipocytokines, adipose tissue and its relationship with cells of the immune system. Gac Med Mex 141(6): 505-512.
11. Charrière G, Cousin B, Arnaud E, André M, Bacou F, Pénicaud L, et al (2003) Preadipocyte conversion to macrophage Evidence of plasticity. J Biol Chem 278(11): 9850-9855.

12. Cannon B, Nedergaard J (2004) Brown adipose tissue: function and physiological significance. Physiol Rev 84(1): 277-359.

13. Rosenbaum M, Pietrobelli A, Vasselli J, Heymsfield S, Leibel R (2001) Sexual dimorphism in circulating leptin concentrations is not accounted for by differences in adipose tissue distribution. Int J Obes Relat Metab Disord 25(9): 1365-1371.

14. Hajer GR, van Haeften TW, Visseren FL (2008) Adipose tissue dysfunction in obesity, diabetes, and vascular diseases. Eur Heart J 29(24): 29592971.

15. Rosen BS, Cook KS, Yaglom J, Groves DL, Volanakis JE, et al. (1989) Adipsin and complement factor D activity: an immune-related defect in obesity. Science 244(4911): 1483-1487.

16. Catalán V, Gómez-Ambrosi J, Rodríguez A, Frühbeck G (2013) Adipose tissue immunity and cancer. Front Physiol 4(275): 1-13.

17. Pond CM (2011) Ecology of storage and allocation of resources: animals. eLS 1.

18. Hotamisligil GS, Shargill NS, Spiegelman BM (1993) Adipose Expression of Tumor Necrosis Factor: Direct Role in Obesity-Linked Insulin Resistance. Science 259(5091): 87-91.

19. Veigas L, Pereira PC, Vicente F, Mesquita MF (2012) Overweight, obesity and abdominal adiposity effects in inflammatory proteins: c-reactive protein and fibrinogen. J Med Sci 12(3): 70-77.

20. Trayhurn P, Alomar SY (2015) Oxygen deprivation and the cellular response to hypoxia in adipocytes-perspectives on white and brown adipose tissues in obesity. Front Endocrinol 6(19): 1-8.

21. Frühbeck G, Gómez-Ambrosi J, Muruzábal FJ, Burrell MA (2001) The adipocyte: a model for integration of endocrine and metabolic signaling in energy metabolism regulation. Am J Physiol Endocrinol Metab 280(6): E827-E847.

22. Fantuzzi G (2005) Adipose tissue, adipokines, and inflammation. J Allergy Clin Immunol 115(5): 911-919.

23. Hotamisligil GS, Budavari A, Murray D, Spiegelman BM (1994) Reduced tyrosine kinase activity of the insulin receptor in obesity-diabetes. Central role of tumor necrosis factor-alpha. J Clin Invest 94(4): 15431549 .

24. Lastra G, Manrique CM, Hayden MR (2006) The Role of $\beta$-Cell Dysfunction in the Cardiometabolic Syndrome. J Cardiometab Syndr 1(1): 41-46.

25. Bharadwaj D, Stein M-P, Volzer M, Mold C, Du Clos TW (1999) The major receptor for C-reactive protein on leukocytes is $\mathrm{Fc} \gamma$ receptor II. J Exp Med 190(4): 585-590.

26. Festa A, D’Agostino R, Howard G, Mykkänen L, Tracy RP, Haffner SM (2000) Chronic subclinical inflammation as part of the insulin resistance syndrome the Insulin Resistance Atherosclerosis Study (IRAS). Circulation 102(1): 42-47.

27. Reseland JE, Anderssen SA, Solvoll K, Hjermann I, Urdal P, et al. (2001) Effect of long-term changes in diet and exercise on plasma leptin concentrations. Am J Clin Nutr 73(2): 240-245.

28. Friedman JM, Halaas JL (1998) Leptin and the regulation of body weight in mammals. Nature 395(6704): 763-770.

29. Sandoval DA, Davis SN (2003) Leptin: metabolic control and regulation. J Diabetes Complications 17(2): 108-113.

30. Farooqi IS, Matarese G, Lord GM, Keogh JM, Lawrence E, et al. (2002) Beneficial effects of leptin on obesity, T cell hyporesponsiveness, and neuroendocrine/metabolic dysfunction of human congenital leptin deficiency. J Clin Invest 110(8): 1093-1103. 
31. Elmquist JK (2001) Hypothalamic pathways underlying the endocrine autonomic, and behavioral effects of leptin. Physiol Behav 74(4): 703708.

32. Cachofeiro V, Miana M, Martín-Fernández B, Heras N, Lahera V (2006) Obesity, inflammation and endothelial dysfunction. Rev Esp Obes 4(4) 195-204.

33. Hirata LL, Sato MEO, Santos CAdM (2004) Free radicals and skin aging Acta Farm Bonaer 23(3): 418-424.

34. Yang R, Barouch LA (2007) Leptin signaling and obesity cardiovascular consequences. Circ Res 101(6): 545-559.

35. Shimomura I, Funahasm T, Takahashi M, Maeda K, Kotani K, et al. (1996) Enhanced expression of PAI-1 in visceral fat: Possible contributor to vascular disease in obeisty. Nat Med 2(7): 800-803.

36. Maruyoshi H, Kojima S, Funahashi T, Miyamoto S, Hokamaki J, et al. (2004) Adiponectin is inversely related to plasminogen activator inhibitor type 1 in patients with stable exertional angina. Thromb Haemost 91(5): 1026-1030.

37. Dulloo AG, Jacquet J, Solinas G, Montani J-P, Schutz Y (2010) Body composition phenotypes in pathways to obesity and the metabolic syndrome. Int J Obes 34 (2): S4-S17.

38. Scherer PE, Williams S, Fogliano M, Baldini G, Lodish HF (1995) A novel serum protein similar to $\mathrm{C} 1 \mathrm{q}$, produced exclusively in adipocytes. J Biol Chem 270(45): 26746-26749.

39. Hu E, Liang P, Spiegelman BM (1996) AdipoQ is a novel adipose-specific gene dysregulated in obesity. J Biol Chem 271(18): 10697-10703.

40. Stumvoll M, Tschritter O, Fritsche A, Staiger H, Renn W, et al. (2002) Association of the TG Polymorphism in adiponectin (Exon 2) with obesity and insulin sensitivity interaction with family history of type 2 diabetes. Diabetes 51(1): 37-41.

41. Ouedraogo R, Gong Y, Berzins B, Wu X, Mahadev K, et al. (2007) Adiponectin deficiency increases leukocyte-endothelium interactions via upregulation of endothelial cell adhesion molecules in vivo. J Clin Invest 117(6): 1718-1726.

42. Fernández-Sánchez A, Madrigal-Santillán E, Bautista M, Esquivel-Soto J, Morales-González Á, et al. (2011) Inflammation, oxidative stress, and obesity. Int J Mol Sci 12(5): 3117-32.

43. Steppan CM, Bailey ST, Bhat S, Brown EJ, Banerjee RR, et al. (2001) The hormone resistin links obesity to diabetes. Nature 409(6818): 307-312.

44. Halliwell B (1994) Free radicals and antioxidants: a personal view. Nutr Rev 52(8): 253-265.

45. Anderson D (1996) Antioxidant defences against reactive oxygen species causing genetic and other damage. Mutat Res 350(1): 103-108.

46. Esposito K, Ciotola M, Schisano B, Misso L, Giannetti G, et al. (2006) Oxidative stress in the metabolic syndrome. J Endocrinol Invest 29(9): 791-795.

47. Amirkhizi F, Siassi F, Minaie S, Djalali M, Rahimi A, et al. (2010) Is obesity associated with increased plasma lipid peroxidation and oxidative stress in women? ARYA Atheroscler 2(4): 189-192.
48. Ray R, Shah AM (2005) NADPH oxidase and endothelial cell function. Clin Sci 109(3): 217-226.

49. Pilz S, März W (2008) Free fatty acids as a cardiovascular risk factor. Clin Chem Lab Med 46(4): 429-434

50. Brasier AR, Recinos A, Eledrisi MS (2002) Vascular inflammation and the renin-angiotensin system. Arterioscler Thromb Vasc Biol 22(8): 12571266.

51. Robertson G, Leclercq I, Farrell GC II (2001) Nonalcoholic steatosis and steatohepatitis. II. Cytochrome P-450 enzymes and oxidative stress. Am J Physiol Gastrointest Liver Physiol 281(5): G1135-G1139.

52. Turrens JF (1997) Superoxide production by the mitochondrial respiratory chain. Biosci Rep 17(1): 3-8.

53. Vincent H, Powers S, Stewart D, Shanely R, Demirel H et al. (1999) Obesity is associated with increased myocardial oxidative stress. Int J Obes Relat Metab Disord 23(1): 67-74.

54. Decsi T, Molnár D, Koletzko B (1997) Reduced plasma concentrations of alpha-tocopherol and beta-carotene in obese boys. J Pediatr 130(4): 653-655.

55. Sies H (1993) Strategies of antioxidant defense. Eur J Biochem 215(2): 213-219.

56. Martinez J (2006) Mitochondrial oxidative stress and inflammation: an slalom to obesity and insulin resistance. J Physiol Biochem 62(4): 303306.

57. Yu BP (1994) Cellular defenses against damage from reactive oxygen species. Physiol Rev 74(1): 139-162.

58. Vincent HK, Vincent KR, Bourguignon C, Braith RW (2005) Obesity and postexercise oxidative stress in older women. Med Sci Sports Exerc 37(2): 213-219.

59. Das UN (2007) Acetylcholinesterase and butyrylcholinesterase as possible markers of low-grade systemic inflammation. Med Sci Monit 13(12): RA214-221.

60. Monteiro R, Azevedo I (2010) Chronic inflammation in obesity and the metabolic syndrome. Mediators Inflamm.

61. Hadi HA, Carr CS, Suwaidi JA (2005) Endothelial dysfunction: cardiovascular risk factors, therapy, and outcome. Vasc Health Risk Manag 1(3): 183-198.

62. González-Chávez A, Elizondo-Argueta S, Gutiérrez-Reyes G, LeónPedroza JI (2011) Pathophysiological implications between chronic inflammation and the development of diabetes and obesity. Cir Cir 79(2): 209-216

63. Couillard C, Ruel G, Archer WR, Pomerleau S, Bergeron J, et al. (2005) Circulating levels of oxidative stress markers and endothelial adhesion molecules in men with abdominal obesity. J Clin Endocrinol Metab 90(12): 6454-6459.

64. Galili O, Versari D, Sattler KJ, Olson ML, Mannheim D, et al. (2007) Early experimental obesity is associated with coronary endothelial dysfunction and oxidative stress. Am J Physiol Heart Circ Physiol 292(2): H904-H911. 
Creative Commons Attribution 4.0 International License

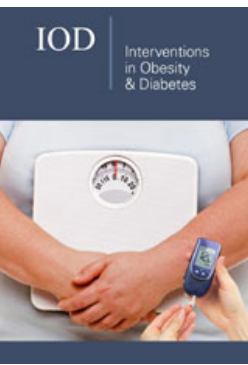

\section{Intervention in Obesity \& Diabetes}

\section{Benefits of Publishing with us}

- High-level peer review and editorial services

- Freely accessible online immediately upon publication

- Authors retain the copyright to their work

- Licensing it under a Creative Commons license

- Visibility through different online platforms 